\title{
Coaching ejecutivo más allá de la relación diádica: el problema del contexto y la cultura organizacionales
}

\author{
Executive coaching beyond the dyadic relationship: \\ the problem of context and organizational culture
}

Sergio Cardona (Universidad Complutense de Madrid, ESIC Business School), Juan Carlos Revilla (Universidad Complutense de Madrid, Instituto TRANSOC) y María T. Arqueros (Universidad Complutense de Madrid)

Artículo recibido: 23 de febrero de 2021 Solicitud de revisión: 25 de febrero de 2021 Artículo aceptado: 3 de junio de 2021

Cardona, Sergio, Revilla, Juan Carlos y Arqueros, María T. (2021). Coaching ejecutivo más allá de la relación diádica: el problema del contexto y la cultura organizacionales. Recerca. Revista de Pensament $i$ Análisi. doi: 10.6035/recerca.5780. Publicación en avance.

\section{Resumen}

El coaching ejecutivo ha dado lugar a numerosos desarrollos de orientación básicamente prescriptiva. Una perspectiva crítica y con base empírica puede permitir ir más allá de la retórica del coaching e identificar las tensiones y las contradicciones de los procesos de coaching en un contexto organizacional, como entorno relacional y cultural (simbólico). El objetivo de este trabajo es acercarnos críticamente al coaching ejecutivo individual en la práctica, estableciendo las diferencias entre lo preconizado por las teorías y los expertos del coaching y lo que se hace en las empresas. Mediante 50 entrevistas en profundidad a coaches y coachees, identificamos cuatro grandes discursos sobre el coaching ejecutivo: $a$ ) herramienta de crecimiento personal; $b$ ) relación diádica; c) resolución de problemas en equipos de trabajo; y d) mecanismo de cambio organizacional. El análisis muestra las tensiones y las contradicciones ad intra y entre los discursos y, con ello, la importancia de considerar el contexto organizacional.

Palabras clave: coaching ejecutivo, cultura organizacional, estudios críticos del management.

Abstract

Executive coaching has produced many prescriptive developments. A critical, empirically-based perspective may allow to move beyond the rhetoric of coaching and identify the tensions and contradictions at stake when processes of coaching are put in practice in an 
organizational context, which is a relational and cultural (symbolic) environment. The aim of this work is to critically approach individual executive coaching as it is carried out in practice, establishing the differences between coaching theories and experts' discourses and actual practices and experiences of coaching. The analysis of 50 in-depth interviews to coaches and coaches have yielded four main discourses about executive coaching: a) tool of personal growth; b) dyadic relationship; c) conflict resolution in working teams; d) mechanism for organizational change. This analysis shows the tensions and contradictions inside and among the discourses and in this way the importance of considering the organizational context as a whole.

Key Words: executive coaching, organizational culture, Critical Management Studies.

\section{INTRODUCCIÓN}

El coaching como proceso de transformación personal en las organizaciones ha dado lugar a numerosos desarrollos con una orientación básicamente prescriptiva. No es frecuente, especialmente en el contexto español, introducirse en el coaching desde una perspectiva empírica que busque conocer la realidad del coaching en la práctica, menos aún desde una perspectiva crítica. Este tipo de perspectiva, crítica y con base empírica, nos puede permitir ir más allá de la retórica del coaching e identificar las tensiones y las contradicciones de poner en marcha procesos de coaching en un contexto organizacional, entendido como un entorno relacional y cultural (simbólico). En este sentido, el objetivo de este trabajo es acercarnos críticamente al coaching ejecutivo individual tal y como se realiza en las empresas, estableciendo las diferencias entre lo preconizado por las teorías y los expertos del coaching y lo que se hace en las empresas. Para ello, tras una introducción que contextualiza la aparición del coaching en las organizaciones, realizamos una comparación entre las tendencias más importantes en la literatura sobre coaching y los discursos de coaches y coachees a partir de su experiencia con el coaching como forma de mostrar las tensiones entre ambas.

El coaching nace de las reflexiones de Gallwey (1997 [1974]) sobre cómo entrenar a los aficionados a jugar a tenis. En este libro se recogen dos principios que serán clave para el coaching, como técnica individual que fomenta la reflexividad subjetiva: la conversación interna que tiene todo jugador y la intervención mínima del entrenador, construida a base de preguntas, que consigue aumentar la conciencia y los recursos del coachee. John Whitmore (2003 [1992]) traspasa los principios del coaching al mundo de la empresa y, con ello, se va extendiendo durante los años 1990 y 2000 por los diferentes países europeos, 
incluidos Francia (Salman, 2014) o España. Progresivamente, el coaching sufre una expansión muy rápida con múltiples escuelas y combinaciones con otras disciplinas. De esta manera, aparecen el coaching ontológico (Echeverría, 2003), desarrollado en Chile e implantado en toda América Latina, el coaching basado en evidencias (Grant, 2016), con mayor desarrollo en Inglaterra y Australia, así como modelos sistémicos, conductuales y construccionistas, pero también basados en la programación neurolingüística ( $\mathrm{PNL}$ ) o en la terapia breve, entre otras técnicas (véase Arqueros, 2015; Cardona, 2015). También aparecen agrupaciones profesionales que desarrollan el coaching: ICF (International Coaching Federation), Asociación Internacional de Coaches Profesionales, AECOP (Asociación de Coaching Ejecutivo y Organizativo), etc.

Esta pluralidad de escuelas y técnicas nos pone sobre la pista de la dificultad de entender el coaching como una práctica unificada y coherente en cuanto forma de intervención en las organizaciones. El elemento común sería, pues, la relación diádica entre coach y coachee en un contexto organizacional dirigida a transformar al segundo, mejorando sus comportamientos o actitudes en relación con su actividad productiva.

Esta configuración del coaching tiene que ver con su surgimiento en el contexto de las transformaciones que sufrieron las organizaciones en el paso del fordismo al posfordismo, es decir, del estilo burocrático de los procedimientos formales a la flexibilidad y la apertura al cambio (Fernández Rodríguez, 2007). Cambian las demandas de las organizaciones a los trabajadores, que deben ser flexibles y adquirir nuevas habilidades y competencias, así como autonomía, capacidad de iniciativa e innovación y liderazgo (Drucker, 1992). También deben poder centrarse en las personas a su cargo para contribuir a su desarrollo (Bennis, 1989). Con esto, la literatura de administración de empresas ha contribuido a generar una mística de las organizaciones «liberadoras» (Vilallonga y Fernández Aguado, 2005), que rescatan el talento y las capacidades de sus empleados y les permiten aportar toda su iniciativa para mejorar su desempeño.

Esta tendencia a la glorificación del liderazgo y la innovación abre el paso hacia la individualización de ciertas dinámicas organizacionales. El coaching forma parte de esta tendencia como forma en que las organizaciones pueden contribuir a la mejora, individual, de aquellas personas en puestos de responsabilidad o con proyección de desarrollo. Así, este énfasis en la transformación del sujeto encaja perfectamente, y quizá esa sea otra clave de su éxito, con la tendencia a la individualización de las relaciones y los procesos sociales que ha sido señalada por distintos autores como característica de nuestra sociedad 
actual. Asistimos, según estos planteamientos, a una creciente psicologización de las prácticas sociales (Álvarez-Uría, 2005; Gordo y De Vos, 2010), que vacía al sujeto de su contenido social y político y lo reemplaza por una preocupación narcisista por sí mismo (Illouz, 2008). Esta psicologización se caracteriza por una sobreatribución individual (interna) y sobreinterpretación psicológica (Rodríguez, 2016), que convierte los problemas sociales en déficits personales (Crespo, Revilla y Serrano, 2006), al tiempo que promete la felicidad a las personas que se ajusten a los estándares que ofrecen las técnicas psi como los libros de ayuda o el propio coaching (Cabanas e Illouz, 2019).

De esta forma, el coaching se convierte en un instrumento de una psicologización política del trabajo (Crespo, Revilla y Serrano, 2006), que se incorpora a unos discursos de management en los que se culpa a la víctima (Fernández Rodríguez, 2007), y no a la organización o al sistema, de un modo de trabajo que, en aras de la eficiencia y la productividad, invade la vida íntima de los trabajadores a través de la figura del coach (Boltanski y Chiapello, 1999) y de un control normativo que busca controlar las mentes y los corazones de los empleados (Kunda, 1995), así como sus emociones (Hochschild, 1983). Con esto, los dispositivos psicologizantes organizacionales, y entre ellos el coaching, se convierten en mecanismos de regulación de las subjetividades de los sujetos laborales, en tecnologías del yo (Foucault, 1996) que buscan la normalización, el ajuste autoproducido (autocontrol, autovigilancia) a unos estándares creados desde la lógica empresarial y que encajan con el orden neoliberal dominante (Rodríguez, 2016). Con ello, las compañías tratan de cultivar «sujetos emprendedores, esto es, individuos autónomos, autorregulados y productivos» (Du Gay, 1996: 60) o, dicho de otro modo, empresarios de sí mismos.

Sin embargo, no deja de existir una cierta inconcreción significativa en cuanto a los destinatarios del coaching. El coaching ejecutivo emerge como una necesidad ante los conflictos tanto laborales como personales de los directivos que requerían de un profesional que les acompañara en su proceso de transformación (Olson, 2008), para establecerse más adelante en los mandos intermedios, como consecuencia de los cambios producidos, en busca de una mejora de la efectividad, productividad y competitividad (Kampa-Kokesch y Anderson, 2001). Pero si el objetivo es fomentar la innovación y el liderazgo carismático, la intervención se habría de dirigir solamente a los directivos de máximo nivel. A pesar de ello, muchos desarrollos sobre coaching lo extienden casi a cualquier trabajador, al menos a todos aquellos que necesiten de autonomía y aprendizajes. No obstante, estas posiciones, digamos, intermedias, están más insertas en la trama de relaciones y dinámicas organizacionales, por 
lo que el contexto y la cultura de la organización son elementos que han de estar más presentes en cualquier intento de intervención sobre ellos.

El carácter del coaching como técnica individualizante convierte la presencia del contexto y la cultura organizacional es un terreno ciertamente en disputa. Mientras algunos teóricos del coaching han señalado su importancia, otros tienden a obviarlos, enfatizando la importancia de la transformación individual. Sin embargo, es posible que parte de los problemas del coachee tengan que ver con unos parámetros de actuación que no encajan con el momento actual de su organización. De ahí la importancia de considerar el contexto y la cultura organizacionales para tomar conciencia sobre los posibles desafíos y oportunidades a los que se enfrenta el coachee (Rosinski, 2008). Esto encajaría también mejor con propuestas que enfatizan lo colectivo en las intervenciones organizacionales, que superan, por tanto, el nivel individual en las estrategias de transformación organizacional.

\section{LOS DISCURSOS TEÓRICOS SOBRE EL COACHING}

A pesar de que el coaching se inserta en los procesos de psicologización de la actividad laboral que hemos descrito, la revisión de los discursos sobre el coaching señala una complejidad entre dos posturas generales en las posiciones de teóricos e investigadores del coaching, entre aquellas que obvian el papel de la cultura organizativa y esas otras que la tienen cuenta. Esto tendría relación, según nuestra posición, con el choque con la lógica organizacional de mejora de su funcionamiento y su eficacia. Por ello, apreciamos dos discursos teóricos amplios que denominamos: 1) coaching como intervención sobre la subjetividad individual y 2) coaching como consultoría organizacional. Siendo conscientes de la imposibilidad de ser exhaustivos, la perspectiva discursiva que tomamos nos permite situarnos por encima del nivel de los autores concretos y tratar de clasificar los distintos planteamientos en los términos discursivos señalados.

\subsection{Coaching como intervención sobre la subjetividad individual}

El primer elemento de este discurso sería la reducción del coaching a la relación interpersonal que se establece entre coach y coachee. Así, la propia Asociación Española de Coaching deja fuera el contexto de la organización en su definición: 
El coaching es un proceso de acompañamiento a través del cual, un/a profesional (coach) acompaña a su cliente (coachee) a conseguir un objetivo. Incluso, en ocasiones, a alcanzar una meta que, en un primer momento, su coachee ni siquiera sospechaba (ASESCO, 2018: 13).

En este marco, el coaching define un proceso personalizado y confidencial mediante el cual el/la coach emplea una serie de herramientas que permiten a su clientela efectuar los descubrimientos y gestionar los cambios necesarios para alcanzar las metas que le proporcionen mayor bienestar y plenitud, tanto a nivel personal como profesional (ASESCO, 2018: 18).

Como se puede ver, se pone el acento en la relación entre coachee y coach sin referencia al contexto organizacional. El objetivo se define de forma individual, pues implica lograr cambiar o mejorar algún aspecto del coachee. Además, la segunda cita insiste en el carácter «personalizado y confidencial» del proceso. Es cierto que no se dice de manera explícita estar en contra de tener en cuenta la cultura organizativa o el contexto, pero sí se sientan las bases para entenderlo de una forma meramente interpersonal en la relación diádica entre coach y coachee.

Otras definiciones recientes van en la misma línea, como esta de Birknerová, Birkner y Zbihlejová (2017: 506), en la que se incide sobre la necesidad de desarrollo de habilidades individuales en el coachee:

El coaching es uno de los métodos de desarrollo de habilidades y capacidades de los gerentes. Es un fortalecimiento específico del potencial de desarrollo del individuo y permite maximizar su desempeño.

Muñoz y Díaz (2014: 64) insertan el coaching en las tendencias actuales del liderazgo carismático y transformador, que legitima de algún modo aún más esa perspectiva individualista del coaching:

El coaching maneja explícitamente elementos referentes al sentido de compromiso y el deber ser de los actores en transmitir e inculcar una visión inspiradora hacia ganar, tener un sentido de liderazgo, responsabilidad de aprendizaje, motivación individual, generar desarrollo profesional.

Por tanto, podemos ver cómo el coaching ejecutivo se centra en la parte individual dejando toda la responsabilidad del cambio al trabajo de coachee y coach. 
Por todos estos elementos, parece apropiado denominar a este conjunto de expresiones discurso de gestión de la subjetividad individual. El coaching aparece como una metodología para la transformación de los sujetos, en sus conductas, habilidades y actitudes, a medio camino entre la mejora del desempeño y el cambio identitario: ser una persona nueva que entienda su trabajo y su labor como directivo, incluso su vida personal, de una forma plenamente distinta. Así lo explican los modelos terapéuticos o conductuales (véase Grant, 2001) que se centran en el cambio de comportamiento del coachee con la finalidad de modificar sus creencias irracionales.

Esto se produce, lógicamente, en la relación entre coach y coachee, de forma que este vínculo diádico se sitúa en primer plano. Así, Villavicencio y Ocaña (2017) enfatizan la necesidad de un acuerdo entre ambas partes, donde se establezcan las bases en cuanto a confidencialidad de la información, duración de las sesiones, lugar y técnicas que se van a usar. El acuerdo formaliza el compromiso del coachee con el proceso de cambio, como pieza clave en los eventuales resultados. Igualmente, todo lo que está fuera de la relación diádica, especialmente la organización, tiene poca relevancia. Así, Zuazo (2010: 3) se refiere a la importancia de atender a las personas dejando a las organizaciones en un segundo plano, pues lo importante es atender la subjetividad individual:

el coaching se hace a personas, no a empresas, y en el caso de una organización, antes de ayudar a solucionar un problema de gestión, rendimientos o metas de la misma, es fundamental que él o los individuos sean conscientes de cuáles son sus motivaciones más íntimas.

Es congruente con esto entender el contexto como freno o impulsor de los cambios producidos en la relación interpersonal de coaching. En este sentido, Villavicencio y Ocaña (2017: 98), como única referencia al entorno de la empresa, afirman que «el entorno puede desequilibrar este proceso y generar dependencia del coach». Es una referencia clave en el sentido en que los actores del proceso de coaching, compañeros de trabajo, colaboradores, jefes, pares... dificultan el cambio del coachee porque les produce incertidumbre y confusión.

Otra forma de individualizar el coaching sería la subjetivación del contexto y la cultura organizacionales, entendiendo que el contexto no es algo objetivo, sino la interpretación subjetiva que del mismo hace el coachee. A esto parecería dirigirse la expresión de conciencia organizacional que utiliza Dyck (2017: 50): 
Otra pregunta para el coachee es cómo utilizar sus habilidades de alto nivel de conciencia organizacional que requieren que lea las relaciones clave y entienda la política del lugar de trabajo para aumentar más empatía en sí misma.

Este es un ejemplo de una autora que no da importancia a la cultura de la organización, aunque aparece de manera indirecta al señalar la necesidad de tener una firme conciencia organizacional: tener en cuenta los problemas de la organización, así como las relaciones interpersonales internas. Por ello, tiende a subjetivizar un concepto claramente social y colectivo como la cultura organizativa, que resulta difícil entender como la interpretación de una persona, siendo más bien el resultado de la forma de hacer y de pensar en una organización a lo largo del tiempo.

\subsection{Coaching como consultoría organizacional}

Para muchos autores, como Rosinski (2008), MacKie (2007), Tooth, Nielsen y Armstrong (2013), Kombarakaran, Yang, Baker y Fernandes (2008), Barner y Higgins (2007), Passmore (2010), McEnroe (2008) o Gallwey (2000), entre otros, la cultura organizativa es clave para el desarrollo del coaching. El coach debe conocer la cultura y el contexto organizacionales como soporte de su desarrollo, de modo que los resultados no solo se visualizan en el individuo, sino en toda la organización. Esto amplía la visión del proceso de coaching y encaja mejor en una concepción, y un discurso, del coaching como consultoría organizacional, que busca casi más la transformación de la organización que la del coachee, aunque esta última sea necesaria para alcanzar la primera.

Las bases de esta concepción del coaching como consultoría se encuentran en muchos desarrollos de corte psicosocial y sociológico (o psicosociológicos; Cardona, 2016), como el enfoque construccionista social o sistémico (Barner y Higgins, 2007), que muestran la inherente sociabilidad del ser humano, la interrelación entre este y el medio cultural y organizacional y la importancia de la interacción social para el comportamiento humano. De este modo, los cambios del sistema repercuten también en los cambios del ejecutivo o coachee. Por este motivo los coaches del management empresarial son parte de estos modelos. Vamos a desarrollar estas cuestiones.

En primer lugar, tanto coach como coachee deben tener en cuenta el contexto social en el que están insertos. Así, Kombarakaran, Yang, Baker y Fernandes (2008) señalan la importancia, para el coaching, del compromiso organizacional del ejecutivo (coachee), del mismo modo que Vidal-Salazar, 
Cordón-Pozo y Ferrón-Vilches (2011: 86) enfatizan la necesidad de que el coach «posea experiencia contrastada como directivo para poder realizar la función de guía profesional de otros directivos». Gray, Ekinci y Goregaokar (2011) senalaron que los conocimientos sobre la estrategia, las habilidades de gestión, la comunicación y la ética organizacional son competencias fundamentales para que cada coach pueda avanzar en la relación. Es decir, tanto coach como coachee no pueden actuar si no es con responsabilidad sobre y conocimiento del contexto organizacional. Es posible que haberse encontrado en situaciones semejantes a las que el coachee se encuentre ayude al coach a entender por lo que está pasando su entrevistado, aunque tendrá que ser consciente también de las diferencias en la situación y el contexto.

En segundo lugar, es clave prestar atención a los vínculos sociales que rodean al coachee en la organización de la que forma parte. Para Barner y Higgins (2007), es necesario tener en cuenta las interacciones sociales del clientecoachee, debido a lo cual el coach ha de acercarse a los otros individuos claves de la organización. Kombarakaran, Yang, Baker y Fernandes (2008) señalan la importancia de un buen programa de apoyo en el entorno organizacional, de las personas que trabajan para o con el coachee, pues forman parte también de la intervención del coach. De hecho, los estudios clásicos sobre el cambio organizacional desde una perspectiva sistémica ya señalaban que una de las dificultades del cambio individual es el propio contexto, pues tiende a reforzar las pautas previas establecidas (Katz y Kahn, 1977).

Especialmente importante es la participación, el apoyo y la implicación de los jefes directos del coachee (Kombarakaran, Yang, Baker y Fernandes, 2008; Dagley, 2010). Los jefes del coachee pueden tener un rol muy distinto, como dar feedback al coach y al coachee o entrevistarse con este después de cada sesión. Por otro lado, los jefes y las jefas del coachee, probablemente, serán representativos de la cultura empresarial en sus valores y criterios para evaluar el desempeño.

En tercer lugar, es necesario considerar la cultura organizacional (Rosinski, 2008), en cuanto que esta influye sobre el coaching, por cuanto que marca los significados que se manejan en el proceso. MacKie (2007) afirma que el coaching puede fallar por un desconocimiento de la cultura de la organización. Además de la cultura de la organización, habría que tener en cuenta la del país en el que se encuentra el coachee, como señala Useche (2004), pues lo que en un entorno cultural puede ser correcto, puede no serlo tanto en otro o ser contraproducente. En este sentido, serían pertinentes los estudios de Hofstede 
(1999) sobre las diferencias culturales entre países, tanto en términos individuales como tipológicos.

Por último, la organización suele estar involucrada en el diseño y el desarrollo de los programas de coaching y sus objetivos no se limitan a lo individual. Así, Vidal-Salazar, Cordón-Pozo y Ferrón-Vilches (2011) entienden que el coach debe conocer en profundidad la estrategia de la empresa y orientarse hacia los objetivos organizativos. Barner y Higgins (2007) sugieren que los coaches deben equilibrar las expectativas de la organización con las metas y las preocupaciones de los coachees. Por tanto, el coaching tiene la particularidad de tener un doble-cliente y la necesidad de producir resultados para ambos (Dagley, 2010), lo que puede ser poco compatible, incluso conflictivo. De hecho, la tendencia en los últimos años va en la dirección de aumentar el contexto del coaching, incluyendo a todos los stakeholders implicados (Burger y Van CollerPeter, 2019).

\section{METODOLOGÍA}

Los presentes análisis se basan en dos investigaciones (Arqueros, 2015; Cardona, 2015) sobre coaching realizadas por los autores de este trabajo, que se presentaron como tesis doctorales en el año 2015. Los doctorandos tenían ya en ese momento experiencia como coaches en distintos tipos de organizaciones, sobre todo empresas. Las dos tesis buscaban conocer cómo se realizan en realidad los procesos de coaching y así observar la eventual existencia de discrepancias entre las teorías de coaching y lo que se podía observar en la experiencia real propia o de otros coaches. Además de una dimensión crítica, presente en este trabajo, ambas tesis se preocupaban también de cómo aumentar la eficacia de los procesos de coaching. Ambos análisis son complementarios e incorporan la necesidad de tener en cuenta el contexto y la cultura dentro de la organización para mejorar los resultados del coaching.

En total se realizaron entrevistas en profundidad entre 2014 y 2015 a cincuenta personas: veinticinco coaches (18 hombres y 7 mujeres) y veinticinco coachees (14 hombres y 11 mujeres). Sin pretender representatividad en esta muestra cualitativa, estas diferencias de género recogen lo que se puede encontrar en el contexto del coaching, con una mayor igualdad de género entre los posibles coachees que entre los coaches, estos últimos de mayor edad en general. De hecho, la media de edad entre los coachees es de 41,3 años (media de hombres $=42$; media de mujeres $=39,8$ ) y la de los coaches es de 53,4 años (media de 
hombres $=56,1 ;$ media de mujeres $=46,4$ ). Por otro lado, se buscó una diferenciación en las orientaciones y experiencia en el caso de los coaches y de tipo de puesto y departamento en el caso de los coachees. Se buscaron diferentes entradas a la contactación para facilitar la diversidad de los perfiles, que se complementó con la estrategia de la bola de nieve. Las entrevistas tuvieron una duración aproximada de una hora y fueron transcritas en su totalidad.

El guion de las entrevistas era flexible y adaptado a la situación, pero se trataron los tipos de coaching, los actores implicados, las orientaciones hacia el coaching, el proceso en sí y sus resultados. Este tipo de entrevistas se han revelado como una técnica de investigación perfectamente adaptada a un área de investigación emergente y que permite acceder a los entresijos de la relación entre coaches y coachees. Las entrevistas de los coachees han sido de tipo narrativo, pues nos cuentan lo que hacen sin tratar de justificar sus prácticas. En el caso de los coaches, las entrevistas son más discusivas. Aparecen más argumentos que razonan, por ejemplo, por qué funciona o no el coaching, lo que nos ha permitido otro tipo de análisis.

Para analizar las entrevistas se utilizaron dos marcos generales: la perspectiva del análisis de la interacción social desde la psicología social y el análisis de discurso (Potter y Wetherell, 1987). El análisis trata de conjugar la comprensión de los procesos de coaching tal como se desarrollan en las organizaciones, con el sentido que tienen para los actores involucrados, y una perspectiva crítica que busca encontrar las contradicciones, las paradojas o las tensiones de las prácticas sociales, inspirada por los estudios críticos del management ${ }^{1}$ (véase Fernández Rodríguez y Medina-Vicent, 2017; Gantman, 2017).

\section{RESULTADOS}

Como resultado del análisis de discursos sobre el coaching, vamos a distinguir cuatro discursos, que desarrollamos a continuación, coaching como: a) herramienta de crecimiento personal, b) relación diádica, c) resolución de problemas en equipos de trabajo y $d$ ) mecanismo de cambio organizacional. En cada discurso trataremos de describir sus ejes principales, sus funciones, cómo es utilizado de forma diferenciada por coaches y coachees y cuáles son sus con-

Los estudios críticos del management son una corriente crítica dentro de los estudios organizacionales que se desarrolla en los años noventa en el Reino Unido que, influida por el posestructuralismo y la teoría del proceso de trabajo, ha tratado de dar cuenta de los procesos de regulación de la subjetividad desde una perspectiva emancipatoria (véase Fernández Rodríguez, 2010) 
tradicciones o paradojas en relación con el carácter individual u organizacional del coaching.

\subsection{Coaching como herramienta de crecimiento personal}

Algunos discursos sobre el coaching enfatizan su papel en el desarrollo profesional de los directivos, ya que permite mejorar sus competencias y habilidades en diferentes ámbitos. Los coaches inciden en la necesidad de ajustarse a las necesidades del coachee y a los resultados que debe conseguir: «...el proceso de coaching está para que el coachee consiga sus resultados» (E18, coach, hombre, 50). Por ello, el coaching se tiene que adaptar a las características de la persona: «...cada persona vive una peripecia distinta, por tanto, eh... entiendo que no tiene sentido que la persona se adapte a un modelo puro de coaching» (E15, coach, hombre, 64). Incluso en las sesiones se da cierta autonomía para que el coachee pueda proponer los temas que se van a tratar: «el coachee va proponiendo temas en los que quiere trabajar» (E7, coach, hombre, 66).

Resulta llamativo que las metas del coaching para el coachee se extiendan en el discurso de los coaches más allá de lo profesional, de forma que alcancen la vida personal: «...van a conseguir aquellos objetivos que quieren en su vida personal y en su vida profesional, y van a sentir que están triunfando...» (E22, coach, hombre, 56). Pero esto es algo que encontramos también en algunas manifestaciones de los coachees, que se apropian de este modo en lo personal de ese desarrollo profesional:

[Necesidades] profesionales y personales, las dos cosas... porque en aquel momento de mi situación... eh... yo detecté una necesidad personal que hasta entonces no la tenía detectada y la estaba ignorando... Entonces... pues... en ese momento, gracias al proceso del coach... que pude... digamos... aceptar que tenía quizás esta necesidad... (E2, coachee, mujer, 36).

Esta apropiación personal del coaching resulta llamativa porque todos son conscientes de que la demanda surge del contexto organizacional: «la empresa es la que paga. Es el cliente. Hay unos objetivos. Hay un marco y el marco lo establece la empresa» (E8, coach, hombre, ${ }_{67}$ ). La compañía entiende que hay necesidad de coaching por cuestiones tan claramente organizativas como el cambio de las funciones asignadas a un directivo o los resultados de las evaluaciones internas del directivo: 
Es un programa [...] dirigido a un colectivo concreto de la organización que son los directivos. Bien que acaban de llegar a la función [...]. O bien que son personas que ocupan este cargo directivo, pero que tienen algún gap en su evaluación (E6, coach, mujer, 45).

Eso sí, también encontramos algún coachee que, digamos, se autoprescribe el coaching ante la circunstancia de cambiar de puesto en la organización, lo cual es buena muestra de la implantación que está alcanzando como técnica de intervención:

Cambié de funciones dentro de la empresa en la que estoy y me encontré con una situación que no sabía muy bien cómo resolverla y contacté con una persona que conozco mucho de la infancia y que actualmente es coach (E2, coachee, mujer, 35$)$.

Por todo ello, en este discurso encontramos una tensión entre unas demandas que suelen provenir de la organización, por tanto orientadas hacia lo profesional, y una tendencia a individualizarlo y remitirlo a lo personal. En el caso de los coaches, es una forma de atender mejor a aquellos con los que trata directamente, aunque no sean el cliente real. Para los coachees, es una forma de apropiación subjetiva del coaching, si bien esto no sucede siempre.

\subsection{Coaching como relación diádica}

Un segundo grupo de discursos se centra en la relación diádica entre coach y coachee, de forma que el trabajo del coach podría entenderse como una técnica, como unos aprendizajes y prácticas que el coach habría de transmitir al coachee y este hacer suyos. Así, la clave sería el logro de cambios en las conductas de los coachees que se pueden entender como objetivos a corto plazo, pues no buscan cambios en estructuras profundas:

...creo que pone un énfasis, el énfasis en los resultados inmediatos. Se centra más en conseguir cosas, no tanto en cambiar estructuras profundas, sino en conseguir cambios... (E1, coach, hombre, 54 ).

En otros casos, los cambios se sitúan en el plano de la organización, como cambios conductuales que suponen un desarrollo de competencias relevantes para el desempeño de sus funciones en la organización: 
...lo que buscas es el desarrollo de hábitos [...]. Llevado al mundo empresarial, esos hábitos se denominan competencias, competencias profesionales y competencias gerenciales o directivas... (E17, coach, hombre, ${ }^{1}$ ).

Estos cambios conductuales suelen referirse a la relación con los compañeros de trabajo, especialmente subordinados del directivo-coachee, como puede ser mejorar la escucha a los subordinados:

Vamos a ver las técnicas para aprender a escuchar... Me he quedado sorprendida [con énfasis]... Lo aplico día a día... Es... es increíble... y cómo la gente también agradece, por supuesto, que le estés escuchando (E2, coachee, mujer, 35).

Por otro lado, también hay una comprensión de que puede ser necesario actuar previamente sobre aspectos cognitivos del sujeto para cambiar la forma de ver el mundo, reformulando situaciones para que se afronten de maneras distintas y los cambios puedan ser más duraderos: «si tú no cambias la manera en que tú ves tu mundo, no hay un cambio sostenible ni sostenido de comportamiento» (E25, coach, hombre, 46 ). Por ello, los coaches enfatizan la necesidad de conocer la percepción o vivencia del contexto de los coachees, como forma de intervenir posteriormente sobre ello y eventualmente modificarlo: «Lo que a mí me interesa es cómo viven ellos eso» (E11, coach, mujer, 43 ).

De esa manera, dan prioridad a la persona como ente individual ante un contexto que no es lo fundamental. La persona es la clave y, por ello, tampoco importa tanto la cultura empresarial en sí como la forma en que la vive el coachee. Por ello, no interesa si se trata de una cultura que facilita o dificulta determinadas acciones que el coachee podría poner en marcha: «la cultura, la cultura, lo que signifique para mi coachee, para mi cliente...» (E7, coach, hombre, 66).

En la misma línea, se enfatiza la necesidad de atender las necesidades del coachee en todas sus dimensiones, incluyendo cuestiones privadas, emocionales, por su impacto sobre los perseguidos cambios cognitivo-conductuales.

...el proceso de coaching se convierte en un acto intimista con los directivos, es decir, es una verdadera puesta en desnudo de lo que siente, de sus angustias, de sus inquietudes, de sus necesidades... [...] Lo que las personas quieren es ser más felices [...] desde el equilibrio de su vida personal y profesional (E22, coach, hombre, ${ }_{56}^{6}$ ).

Por ello, para que la relación funcione, es necesario que el coachee entre en el juego, se implique y sea capaz de exponerse, incluso en su intimidad, ante 
una persona en principio desconocida y en un marco que no suele venir definido por el propio coachee, sino, como hemos comentado, por la organización: «...yo te voy a acompañar para que tú des esos pasos que necesitas para estar más feliz, pero yo no sé cómo hacerte más feliz, si tú no me dices lo que te hace feliz» (E22, coach, hombre, 56).

Lo que para el coach, normalmente con experiencia, es algo que va de suyo, para el coachee no es tan evidente, ni esperado, que el coaching tenga que tener una implicación tan grande en la intimidad personal, que obliga a expresar sentimientos en un contexto en principio profesional:

Al final fuimos concretizando... Partiendo de un marco muy general y a lo mejor muy emocional, y muy mezclado con lo personal [...] No tuve ningún problema, pero era un tema que no me esperaba (E2, coachee, mujer, 35).

Para los coaches, esta implicación personal del coachee tiene que ver con su actitud, la química en la relación, la confianza, el compromiso, la responsabilidad, etc.: «aquella persona que viene al coaching con buenas actitudes y buenas expectativas, y que se abre al proceso y que pone encima de la mesa la voluntad de hacer cambios...» (E1, coach, hombre, 54 ); «...es muy importante que esa química exista, muy importante» (E13, coach, mujer, 45).

El problema, desde nuestro punto de vista, es que se está pidiendo una colaboración muy personal en un proceso que no es autodeterminado, sino enraizado en una lógica organizacional. Cierto es que los receptores del coaching se pueden ver como privilegiados, porque la organización ha pensado en ellos como merecedores de coaching, normalmente por sus posibilidades de proyección personal; y que, si el coach es externo, la colaboración pueda ser más fácil. Aun así, como proceso externo, el coachee puede tener dudas sobre las consecuencias que podría tener para él o ella, que no necesariamente tendrían que ser positivas: «...lo que pasa que al final estás metida en un proceso que ha encargado tu jefe y que... no sabes de alguna manera en qué se traduce eso» (E17, coachee, mujer, 54).

Por ello, el coaching puede generar resistencias. Para el coach se puede entender como falta de colaboración o de actitud positiva. Pero para el coachee se podría ver como mecanismos de autoprotección ante dinámica ajenas. Aunque son pocas las instancias discursivas de resistencias que encontramos, puede ser interesante mostrar dos estrategias de distanciamiento respecto del coaching que podemos entender como resistencia oculta o acomodación crítica (véase Revilla, 2017). En primer lugar, el coaching se puede entender como algo 
irrelevante, una herramienta sin un objetivo o unos resultados claros: «quedará como una cosa más de uno de tantos procesos que se hacen y que sirven para poco... y que se hacen por rutina en las empresas» (E2o, coachee, hombre, 42).

En segundo lugar, el coachee puede poner límites a la labor del coach. Se puede limitar la implicación emocional, el rango de temas que se van a tratar o, como en el siguiente verbatim, el conocimiento del coach del contexto organizacional y las situaciones concretas por las que atraviesa el coachee. En el siguiente verbatim el coachee se sitúa en una posición de decidir dónde puede entrar o no el coach, además de entender que todo lo que está fuera de la relación diádica no es relevante para la intervención sobre el coachee, pues supone inmiscuirse en su espacio propio:

...tampoco es su cometido el tomar parte, y el de entender bien la situación [...]. Yo creo que no es importante saber del trabajo, ni conocer la situación... sino que lo importante es lo que le está pasando a esa persona en sus procesos mentales ... (E24, coachee, hombre, 46).

En definitiva, este segundo discurso entra en una cierta contradicción, también, con la presencia de las relaciones del coachee con sus compañeros de trabajo, así como con la determinación organizacional de los programas de coaching, al tiempo que impone una implicación emocional que puede sentirse como violenta. Ambos elementos inciden necesariamente sobre la relación diádica, condicionando tanto las sesiones de coaching, que deben tratar las situaciones vividas por el coachee en su puesto de trabajo, como los propios objetivos de los cambios que se van a producir en el sujeto, que están al menos en parte predeterminados por la demanda organizacional.

\subsection{Coaching como resolución de conflictos en equipos de trabajo}

Este grupo de discursos se encuentra especialmente cuando coaches, pero sobre todo coachees, describen con detalle las cuestiones que se trabajan en las sesiones de coaching. Se aprecia con claridad que las preocupaciones de los coachees se dirigen a las relaciones con sus subordinados o con sus superiores o, en general, con la gestión o dirección de equipos.

Era un problema de relación... integración con una persona con la que yo tenía que trabajar mucho... con la cual no nos entendíamos y... bueno... pues teníamos un montón de roces profesionales ( $\mathrm{E}_{5}$, coachee, hombre, ${ }_{41}$ ). 
Por ello, la actuación del coach se dirige a ayudar a resolver eso que se vive como problema, lo que puede requerir de un conocimiento, incluso directo, de todo el contexto laboral del coachee, lo cual es destacado por muchos coaches, sobre todo cuando la demanda se genera desde la propia organización.

Alguna vez le he pedido a algún directivo que me permitiese asistir a alguna reunión de sus comités ejecutivos para ver su entorno, su equipo, cómo se mueven, cómo se relacionan, cómo se comunican... (E23, coach, hombre, 54).

De hecho, es frecuente que se dé entrada, directa o indirectamente, al superior del coachee en el proceso de coaching, en forma de sesiones tripartitas o de feedback. En este verbatim se aprecia cómo el coaching supera la relación diádica, pues los cambios que necesita el coachee se negocian con el superior, que participa también en el desarrollo del proceso de coaching mediante estas sesiones tripartitas o con formas de feedback formal o informal:

Tú imagínate que el coachee quiere trabajar un tema con su jefe, entonces esa sesión tripartita es muy potente. Ahí se establece un marco de relación y, en ese marco de relación, el coach declara: «Vale ¿estás de acuerdo en trabajar lo que está visualizando tu jefe?» (E18, coach, hombre, 50 ).

Por otro lado, coaches y coachees inciden sobre la importancia que tiene la formación o los conocimientos de los primeros para hacer el coaching más efectivo. Esto es una forma de indicar también que el coaching está inserto en un contexto organizacional, por lo que los conocimientos y la formación de los coaches en este ámbito se perciben como facilitadores del logro de los objetivos del coaching. Así, puede ser útil la formación en ciencias organizacionales o los conocimientos y los estudios de comportamiento organizacional:

...la formación, todo lo que tiene relación con los comportamientos organizacionales, de los grupos, la dinámica de las relaciones estructurales o roles en los equipos, principios fundamentales del management... (E12, coach, hombre, 68).

Igualmente, la experiencia del coach en organizaciones, sobre todo si han sido distintos tipos de organizaciones y culturas, también se percibe como facilitador de su actuación, pues se conocen las tensiones a las que los profesionales se ven sujetos y se aprecian fenómenos que desde dentro no se observan. 
¿Mi experiencia laboral y profesional? Sí, hombre, claro, sí te sirve. Te sirve en el sentido en que te da un contexto dentro de lo que es el coaching. [...] sí que te da, de alguna manera, cuál es el contexto de las empresas, qué dificultades, qué tipo de relaciones internas se establecen en el ámbito profesional (E5, coach, mujer, 38 ).

En algunos casos se destaca la importancia de que la experiencia del coach se haya producido como directivo, ya que estamos hablando de coaching directivo, para conocer las dinámicas internas de ese mundo:

para mí un coach debe haber sido directivo [...] de cómo funcionan las dinámicas dentro de un comité de dirección, o desde una filial o un departamento... (E25, coach, hombre, 46).

En definitiva, este discurso recoge muy bien la dinámica propia del coaching como proceso en el que están presentes, de diversas maneras, los subordinados, los superiores y los equipos de trabajo. La experiencia organizacional del coach permite la intervención sobre la relación con su equipo de trabajo. Por tanto, permite apreciar su inserción radical en un contexto organizacional. Esto no niega la dimensión diádica e individual del coaching, que hemos reseñado, pero sí permite entenderlo en toda su complejidad.

\subsection{Coaching como mecanismo de cambio organizacional}

Estos discursos remiten al papel del coaching en la transformación o desarrollo de la organización en conjunto. Esta, como cliente último, ha de encajar los programas de coaching directivo en las políticas y los objetivos organizacionales, por mucho que se concreten en personas individuales, que participarán en las sesiones de coaching. Así, si se entienden como una inversión de la compañía, esta debería tener un retorno, como cualquier otra inversión empresarial:

...todas las empresas miran muy bien el retorno de esa inversión y, por lo tanto, ese coaching tiene una trascendencia distinta a la que tiene con un particular... (E24, coach, hombre, 67).

La tensión mayor de este discurso es que, partiendo de ese diseño organizacional, tiene que concretarse en unas actuaciones sobre individuos concretos. Por eso, el coaching tiene que adaptarse a la situación y a las características de los directivos que necesitarían este apoyo externo: 
...las necesidades de los altos ejecutivos son más de cómo tomar decisiones muy complejas que afectan a muchas personas.... Lo que necesitan es tener confianza para tomar ese tipo de decisiones, con lo que el enfoque de coaching es más personal que con el mando intermedio... [...]. Con mandos intermedios lo que me suelen pedir es que aborde temas que tienen que ver con las competencias habituales de liderazgo... (E22, coach, hombre, ${ }_{5}^{6}$ ).

Como se puede apreciar, el salto entre la necesidad originaria de cambio organizacional y la concreción en actuaciones sobre sujetos concretos con necesidades particulares específicas es grande. Incluso se podría plantear si el coaching puede ser la forma más idónea de producir cambios organizacionales, dada su naturaleza individual. De hecho, la práctica total ausencia de esta dimensión organizacional del coaching entre los coachees sería otra muestra de este mencionado salto, de la dificultad de percibir el objetivo de transformación organizacional del coaching directivo.

Los coaches sí son más conscientes de esa dimensión organizacional y también de cómo otras cuestiones organizacionales se cruzan en la relación diádica. Especialmente son interesantes las reflexiones que encontramos sobre la influencia del tipo de cultura organizacional, pues impregna las relaciones que se producen en el seno de la organización, lo que necesariamente afectará al coaching. Por ello, el conocimiento del coach de la cultura organizacional podría ser también interesante a la hora del desarrollo del coaching:

Qué culturas pueden favorecer o no favorecer el proceso de coaching, ¿no? Porque puede pasar que tú estés trabajando con el coaching una serie de temas, pero que se den de narices con la cultura organizativa (E1, coach, hombre, 54$)$.

[El coach] tiene que ser alguien independiente, pero con algo de conocimiento sobre la cultura de la organización (E15, coachee, hombre, 32).

En definitiva, en este caso apreciamos una cierta incongruencia entre unos discursos que enfatizan el encaje organizacional de los procesos de coaching y unas prácticas que terminan por individualizar la intervención en unos determinados directivos de la organización, con lo que se pierde de vista el objetivo organizacional de origen. Por otro lado, la organización, expresada en su cultura propia, tiene una presencia ineludible en el coaching, al que condiciona. 


\section{CONCLUSIONES}

La revisión de la literatura sobre coaching ejecutivo nos permitió identificar dos polos discursivos: el primero, centrado en los aspectos personales e individualistas y, el segundo, inserto en las necesidades y demandas organizacionales. Esta revisión nos ha mostrado que los planteamientos dominantes sobre el coaching exceden los procesos de psicologización en los que se suele enmarcar en la literatura crítica (Salman, 2014; Rodríguez, 2016; Cabanas e Illouz, 2019) y apuntan a una tensión entre la dimensión individual y organizacional del coaching.

De hecho, el análisis de las entrevistas a coaches y coachees nos ha conducido a complejizar aún más la situación del coaching al identificar la relevancia de cuatro discursos que remiten a los diferentes niveles de la experiencia del coaching. Sin embargo, la escasez de trabajos de coaching desde una perspectiva crítica dificulta poner en diálogo los resultados obtenidos con la literatura previa.

El primero de estos discursos sitúa el coaching como herramienta de crecimiento personal, con las necesidades y las capacidades del coachee en el centro y, por ello, sería el discurso más cercano a la idea de la psicologización. Sería un proceso de descubrimiento y de desarrollo personal, casi más que profesional. Esto refleja el carácter del coaching como práctica normalizadora y disciplinaria de las subjetividades (Cushion, 2018). Por ello, se produce una tensión entre el cliente real del coaching, la organización, y el usuario y objetivo del desarrollo del potencial, que es el coachee. En este sentido, se da una cierta apropiación privada del coaching ejecutivo.

El segundo discurso entiende el coaching como relación diádica, colocando la clave en la producción de cambios a corto plazo en la conducta del coachee a partir de las reuniones con su coach, bien trabajando directamente sobre su conducta mediante distintas herramientas, bien mediante modificaciones en sus ideas y modelos mentales. Sin embargo, este discurso parece dejar al margen al resto de la organización, cuando la relación de coaching afectará a su entorno de superiores y colaboradores. Igualmente, el entorno cultural y las demandas de la organización quedan relegados a la interpretación que el coachee hace de este. El concepto clave de este discurso es, pues, la persona como profesional descontextualizado y reducido a una relación diádica entre el coachee y su coach. Este discurso encajaría bien con el análisis de Salman (2014) sobre la contribución del coaching a una mejor gestión de las temporali- 
dades laborales, lo que termina por responsabilizar al directivo de la mala organización de sus tiempos o a cuestiones de personalidad.

Un tercer discurso amplía el foco y sitúa el coaching en una situación de equipo de trabajo, que protagonizará las reuniones de coaching: superiores, colaboradores, pares, clientes, etc.; son actores que se incorporan al coaching como elemento central, de forma que se va más allá de la relación diádica entre coach y coachee (Kombarakaran, Yang, Baker y Fernandes, 2008). No es extraño, por ello, que el jefe participe en el coaching para aportar su visión de los temas y del desempeño de su colaborador. Así, el tema tratado tendrá, al menos, la perspectiva de cada uno de los actores que le dan significado, incluida la cultura organizacional, que también aporta significados. Por ello, este discurso coloca el coaching como herramienta de resolución de conflictos en equipos de trabajo.

Por último, el coaching aparece como mecanismo de cambio organizacional donde el contexto y la cultura organizacional son claves (Rosinski, 2008; Mackie, 2007). Sin embargo, es difícil que este se produzca si no es en un contexto de cambio que afecte de manera significativa a la organización. Por ello, apreciamos cierta tensión entre la dimensión individual y relacional del coaching y el objetivo de conseguir ciertos cambios necesarios en el funcionamiento y en la cultura de la organización.

Con estos análisis, esperamos haber podido poner de manifiesto las tensiones y las contradicciones que existen a la hora de implementar programas de coaching en las organizaciones. Mientras que otras investigaciones han mostrado los efectos disciplinarios sobre las subjetividades de los directivos de prácticas como el coaching (Salman, 2014; Cushion, 2018; Cabanas e Illouz, 2019), la perspectiva propia de este trabajo nos permite apreciar justamente que, incluso desde la lógica de la organización, el coaching presenta grandes limitaciones precisamente por estas contradicciones en su implantación. Así, el coaching, por su orientación individual e interpersonal, presenta un riesgo claro de ignorar el entorno de la organización y quedarse exclusivamente en el campo del coachee o, incluso, poner en marcha acciones negativas para la organización. Por ello, un énfasis desmesurado en una perspectiva en exceso psicológica o individualista para el coaching nos parece contraproducente, de forma que abogamos por una orientación compleja y comprensiva del coaching que reconozca todas sus dimensiones y pueda, de ese modo, producir los objetivos organizacionales deseados. Creemos, en este sentido, necesario seguir investigando la relación entre coaching ejecutivo y cultura organizativa y, a la vez, 
reclamar un papel más activo de esta en los procesos de desarrollo de potencial.

Eso sí, desde una perspectiva más amplia sería coherente cuestionar que desde las organizaciones se busquen ciertas transformaciones en su funcionamiento mediante herramientas como el coaching, que se dirigen directamente al sujeto y solo indirectamente a la organización. En ese sentido, podrían ser más convenientes formas de intervención en las organizaciones orientadas más directamente a la transformación del conjunto, donde el coaching podría ser uno de los programas que se implementen, pero no el único ni el más importante.

\section{BIBLIOGRAFÍA}

Álvarez-Uría, Fernando (2005). Viaje al interior del yo: la psicologización del yo en la sociedad de los individuos. Claves de razón práctica, 153, 61-67.

Arqueros, María T. (2015). El proceso de coaching ejecutivo: evaluación de los factores que determinan su eficacia a través de un análisis de discurso [Tesis doctoral]. Universidad Complutense de Madrid. Recuperado de: https://eprints.ucm.es/37431/1/T37138.pdf [Consultado el 9 de agosto de 2020]

ASESCO, Asociación Española de Coaching (2018). El coaching se hace mayor: el Libro Blanco del Coaching. Almería: Editorial Círculo Rojo. Recuperado de: http://www.asescoaching.org/documentos/ EL\%20LIBRO\%2oBLANCO\%2oDEL\%20COACHING.pdf

[Consultado el 22 de febrero de 2021]

Barner, Robert y Higgins, Julie (2007). Understanding implicit models that guide the coaching process. Journal of Management Development, 26(2), 148-158. Recuperado de: https://www.researchg ate.net/publication/235294484_Understanding_implicit_models_t hat_guide_the_coaching_process

Bennis, Warren G. (1989). Managing the Dream: Leadership in the 21st Century. Journal of Organizational Change Management, 2(1), 6-10.

Birknerová. Zuzana, Birkner, Martin y Zbihlejová, Lucia (2017). Analysis of Links between Communication Skills, Motivational Orientations, and Awareness of Coaching. International Journal of Organizational Leadership, 6, 505-510. Recuperado de: https://ijol.ci 
kd.ca/article_6o381_ae6ad535678040ec7oefo4fce7ec2da1.pdf [Consultado el 22 de febrero de 2021]

Boltanski, Luc y Chiapello, Ève (1999). Le nouvel esprit du capitalisme. Paris: Gallimard.

Burger, Zelda y Van Coller-Peter, Salome (2019). A guiding framework for multi-stakeholder contracting in executive coaching. SA Journal of Human Resource Management, 17, a1114. doi: 10.4102/sajhrm. v17io.1114

Cabanas, Edgar e Illouz, Eva (2019). Happycracia. Cómo la ciencia y la industria de la felicidad controlan nuestras vidas. Barcelona: Planeta Libros.

Cardona, Sergio (2015). Coaching en las organizaciones: una perspectiva desde la Psicología Social [Tesis doctoral]. Universidad Complutense de Madrid. Recuperado de: https://eprints.ucm.es/37905/1/T37279.pdf [Consultado el 9 de julio de 2020]

Cardona, Sergio (2016). Coaching en las organizaciones: una perspectiva desde la Psicología Social. Madrid: ESIC.

Crespo, Eduardo, Revilla, Juan Carlos y Serrano, Amparo (2006). La psicologización política del trabajo. En Dorna, Alejandro (Ed.): Psicología Política. Bogotá: Psicom.

Cushion, Christopher J. (2018). Reflection and reflective practice discourses in coaching: a critical analysis. Sport, Education and Society, 23(1), 82-94.

Dagley, Gavin (2010). Exceptional executive coaches: Practices and attributes. International Coaching Psychology Review, 5(1), 63-80. Recuperado de: http://ocu.mhwilleke.s3.amazonaws.com/2_Except ional\%2oexecutive\%2ocoaches\%2oPractices\%20and\%20attributes.pd $\mathrm{f}$ [Consultado el 22 de febrero de 2021]

Drucker, Peter F. (1992). Managing for the future. The 1990 and Beyond. New York: Truman Tallet Books.

Du Gay, Paul (1996). Consumption and identity at work. London: Sage.

Dyck, Loren R. (2017). Coaching with Distinctive Human Strengths for Intentional Change. Journal of Leadership, Accountability and Ethics, 14(2). Recuperado de: https://www.articlegateway.com/inde x.php/JLAE/article/view/1602

Echeverría, Rafael (2003). Ontología del Lenguaje. Santiago de Chile: Comunicaciones Noroeste. 
Fernández Rodríguez, Carlos J. (2007). El discurso del Management: tiempo y narración. Madrid: Centro de Investigaciones Sociológicas.

Fernández Rodríguez, Carlos J. (ed.) (2010). Vigilar y organizar. Madrid: Siglo XXI.

Fernández Rodríguez, Carlos J. y Medina-Vicent, Maria (2017). Los nuevos discursos del management: difusión, impactos y resistencias. Recerca, 20, 7-14. doi: 10.6035/Recerca.2017.20.1

Foucault, Michel (1996). Tecnologías del yo y otros textos afines. Barcelona: Paidós.

Gallwey, W. Timothy (1997 [1974]). El juego interior del trabajo. Málaga: Sirio.

Gallwey, W. Timothy (200o). El juego interior del trabajo. Concentración, movilidad, aprendizaje y placer en el lugar de trabajo. Madrid: Sirio.

Gantman, Ernesto (2017). En torno al potencial transformador de los CMS (Critical Management Studies). Recerca, 20, 15-33. https://doi.org/10.6035/Recerca.2017.20.2

Gordo, Ángel y De Vos, Jan (2010). Psychologism, Psychologising and De-Psychologisation. Annual Review of Critical Psychology, 8, 3-7.

Grant, Anthony M. (2001). Towards a Psychology of Coaching. The Impact of Coaching on Metacognition, Mental Health and Goal Attainment. Ph.D. Dissertation. Sydney: University of Sydney. Recuperado de: https://www.researchgate.net/publication/228598 134_Towards_a_psychology_of_coaching

Grant, Anthony M. (2016). What constitutes evidence-based coaching? A two-by-two framework for distinguishing strong from weak evidence for coaching. International Journal of Evidence Based Coaching and Mentoring, 14(1), 74-85.

Gray, David E., Ekinci, Yuksel y Goregaokar, Harshita (2011). A five-dimensional model of attributes: Some precursors of executive coach selection. International Journal of Selection and Assessment, 19 (4), 415-428.

Hochschild, Arlie Russell (1983). The managed heart. Berkeley: University of California Press.

Hofstede, Geert (1999). Culturas y organizaciones, el software mental: La cooperación internacional y su importancia para la supervivencia. Madrid: Alianza. 
Illouz, Eva (2008). Saving the Modern Soul: Therapy, Emotions, and the Culture of Self-Help. Berkeley: University of California Press.

Katz, Daniel y Kahn, Robert L. (1977). Psicología Social de las Organizaciones. México: Trillas.

Kampa-Kokesch, Sheila y Anderson, Mary Z. (2001). Executive Coaching. A Comprehensive Review of the Literature. Consulting Psychology Journal: Practice and Research, 53(4), 205-228.

Kombarakaran, Francis A., Yang, Julia A., Baker, Mila N. y Fernandes, Pauline B. (2008). Executive Coaching: It works! Consulting Psychology Journal: Practice and Research, 60(1), 78-90.

Kunda, Gideon (1995). Engineering Culture: Control and Commitment in a High-Tech Corporation. Organization Science, 6(2), 228-230.

MacKie, Doug (2007). Evaluating the effectiveness of executive coaching: Where are we now and where do we need to be? Australian Psychologist, $\quad 42(4), \quad 310-318 . \quad$ doi: 10.1080/00050060701648217

McEnroe, Douglas (2008). Coaching y liderazgo. En Kaufmann, Alicia E. y Cubeiro, Juan Carlos. Coaching y diversidad (117-126). Madrid: LID.

Muñoz Maya, Carlos Mario y Díaz Villamizar, Olga Lucía (2014). El Coaching y la transformación organizacional: una oportunidad para las Empresas y los Coaches. Suma de Negocios, 5(11), 62-69. doi: $\left.10.1016 / S_{2215-910 X(14)}\right)_{0020-5}$

Olson, Paul O. (2008). A review of assumptions in executive coaching. The Coaching Psychologist, 4(3), 151-159. Recuperado de: http://www .pauloolson.com/x/resources/Olson2oo8ExecutiveCoaching.pdf

Passmore, Jonathan (2010). A grounded theory study of the coachee experience: The implications for training and practice in coaching psychology. International Coaching Psychology Review, 5(1), 48-62. Recuperado de: https://www.researchgate.net/publication/ 47529315_A_grounded_theory_study_of_the_coachee_experience_T he_implications_for_training_and_practice_in_coaching_psycholo gy [Consultado el 22 de febrero de 2021]

Potter, Jonathan y Wetherell, Margaret (1987). Discourse and social psychology: beyond attitudes and behaviour. London: Sage. 
Revilla, Juan Carlos (2017). Reconstrucciones de la identidad laboral en contextos organizacionales invivibles. Política y Sociedad, 54(1), 65-86.

Rodríguez, Roberto (2016). La psicología en el proyecto cultural neoliberal: literatura de autoayuda y gestión de subjetividades. En Rodríguez, Roberto (Ed.). Contrapsicología (351-380). Madrid: Dado.

Rosinski, Philippe (2008). Coaching y Cultura: Herramientas para apalancar las diferencias nacionales, corporativas y profesionales. Buenos Aires: Gran Aldea.

Salman, Scarlett (2014). Un coach pour battre la mesure ? La rationalisation des temporalités de travail des managers par la discipline de soi. Revue d'anthropologie des connaissances, 8(1), 97122.

Tooth, Julie-Anne, Nielsen, Sharon y Armstrong, Hilary (2013). Coaching effectiveness survey instruments: taking stock of measuring the immeasurable. Coaching: An International Journal of Theory, Research and Practice, 6(2), 137-151. doi: 10.1080/17521882.2013.802365

Useche, María Cristina (2004). El coaching desde una perspectiva epistemológica. Revista de Ciencias Sociales, 105, 125-132. Recuperado de: https://www.revistacienciassociales.ucr.ac.cr/imag es/revistas/RCS105/o8Useche.pdf

Vidal-Salazar, María Dolores, Cordón-Pozo, Eulogio y FerrónVilches, Vera (2011). Efectividad del coaching en los procesos de mejora de gestión de empresas. Universia Business Review, 31, 82101. Recuperado de: http://www.integraorg.com/wpcontent/uploads/2014/o5/Efectividad-del-coaching.pdf

Vilallonga, Mariano y Fernández Aguado, Javier (2005). Progreso directivo y coaching empresarial. Navarra: Ediciones Internacionales Universitarias.

Villavicencio Álvarez, Víctor Emilio y Ocaña Arguello, Irene Susana (2017). El coaching como herramienta para mitigar la resistencia al proceso de cambio organizacional. Palermo Business Review, 16, 83100.

Whitmore, Timothy (2003 [1992]). Coaching: el método para mejorar el rendimiento de las personas. Barcelona: Paidós. 
Zuazo, Pedro (2010). El coaching: la herramienta del siglo XXI. Madrid: ASESCO. 